\title{
A Graph-Theoretical Approach to Boolean Interpolation of Non-Boolean Functions
}

\author{
Sergiu Rudeanu \\ University of Bucharest \\ Faculty of Mathematics \\ Bucharest, Romania \\ rudefuninf.math.unibuc.ro
}

\author{
Dan A. Simovici \\ Univ. of Massachusetts Boston, \\ Dept. of Comp. Science, \\ 02125 USA \\ dsimecs.umb.edu
}

\begin{abstract}
We introduce a graph-theoretical approach to the study of approximation of non-Boolean functions on Boolean algebra. We show that optimal interpolations of non-Boolean functions by Boolean functions are linked to minimal chromatic decompositions of graphs attached to these functions and we study special vertices in these graphs.
\end{abstract}

\section{Introduction}

The purpose of this paper is to develop a graphtheoretical approach for finding optimal Boolean interpolations of non-Boolean functions on Boolean algebras (see $[10,9]$ ). The interest in non-Boolean function has been sparked by work dealing with the applications of set-valued non-Boolean functions in circuit design $[3,8,1,2,6,5]$ and [4].

Let $\mathcal{B}=\left(B, \vee, \cdot{ }^{\prime}, 0,1\right)$ be a Boolean algebra, where $B$ is a set, $\vee$ and $\cdot$ are binary operations on $B$ called disjunction and conjunction, respectively, ' is a unary operation, called the complementation operation, and 0,1 are two special elements of $B$, with $0 \neq 1$ such that the usual axioms of Boolean algebras are satisfied as given e.g. in [11].

Elements of $B^{n}$, where $n>1$ will be denoted by capital letters $X, Y, \ldots$, while elements of the algebra $\mathcal{B}$ will be denoted by small letters. Elementary $n$-tuples, that is, members of the set $\{0,1\}^{n}$, will be designated by $A, B, \ldots$. Boolean functions will be denoted by small letters $f, g, \ldots$ Arbitrary functions will be denoted by capital letters: $F, G, \ldots$.

If $x \in B$ and $a \in\{0,1\}$ we use the notation

$$
x^{a}= \begin{cases}x & \text { if } a=1 \\ x^{\prime} & \text { if } a=0\end{cases}
$$

Note that $a^{a}=1$ for $a \in\{0,1\}$. If $X=\left(x_{1}, \ldots, x_{n}\right) \in$ $B^{n}$ and $A=\left(a_{1}, \ldots, a_{n}\right) \in\{0,1\}^{n}$, define $X^{A}$ as the conjunction $x_{1}^{a_{1}} \cdots x_{n}^{a_{n}}$. We have

$$
A^{C}= \begin{cases}1 & \text { if } A=C \\ 0 & \text { if } A \neq C,\end{cases}
$$

for every $A, C \in\{0,1\}^{n}$. Also, if $A \neq C$, we have $X^{A} X^{C}=0$ for every $X \in B^{n}$.

The binary operation " + " is defined on $B$ by $x+y=$ $x y^{\prime} \vee x^{\prime} y$ for $x, y \in B$. An easy argument by induction on $n$ shows that if $z_{1}, \ldots, z_{n} \in B$ such that $z_{i} z_{j}=0$ for $1 \leq i, j \leq n$ and $i \neq j$, then

$$
\bigvee_{1 \leq i \leq n} z_{i}=\sum_{1 \leq i \leq n} z_{i}
$$

If $X=\left(x_{1}, \ldots, x_{n}\right) \in B^{n}$, then we denote $x_{1} \vee x_{2} \vee$ $\cdots \vee x_{n}$ by $X^{\mathbf{v}}$. Also, if $X, Y \in B^{n}, X=\left(x_{1}, \ldots, x_{n}\right)$ and $Y=\left(y_{1}, \ldots, y_{n}\right)$, we denote by $X+Y$ the $n$-tuple $\left(x_{1}+y_{1}, \ldots, x_{n}+y_{n}\right)$.

Lemma 1.1 Let $\mathcal{B}=\left(B, \vee, \cdot,{ }^{\prime}, 0,1\right)$ be a Boolean algebra.

1. For every $X \in B^{n}$ we have $\bigvee_{A \in\{0,1\}^{n}} X^{A}=1$.

2. If $X, Y \in B^{n}$, then $1+\bigvee_{A \in\{0,1\}^{n}} X^{A} Y^{A}=(X+$ $Y)^{\mathbf{v}}$.

Proof. Since both identities involve only Boolean operations, they can be proven immediately by MüllerLövenheim Verification Theorem (Theorem 2.13 from [11]) by observing that the functions designated by the expressions from the left and right members are equal for all elementary vectors $C \in\{0,1\}^{n}$.

It is useful to note that the mapping $d: B^{n} \times B^{n} \longrightarrow B$ defined by $d(X, Y)=(X+Y)^{\mathbf{v}}$ for $X, Y \in B^{n}$ is a distance on $B^{n}$, in the sense defined in [11], p. 313. Namely, 
it is clear that $d(X, Y)=0$ if and only if $X=Y$ and that $d(X, Y)=d(Y, X)$. It is easy to verify that for every $x, y, z \in B$ we have

$$
x+y \leq(x+z) \vee(z+y) .
$$

This implies immediately

$$
d(X, Y) \leq d(X, Z) \vee d(Z, Y)
$$

for $X, Y, Z \in B^{n}$, which justifies our observation.

\section{The Graph of a Function over $B^{n}$}

Let $F: B^{n} \longrightarrow B$ be a function. Its graph $\Gamma_{F}$ has $B^{n}$ as its set of vertices; an edge $(X, Y)$ exists in $\Gamma_{F}$ if

$$
F(X)+F(Y) \not \subset(X+Y)^{\vee} .
$$

To simplify the notation we denote the relation defined by the graph $\Gamma_{F}$ by $\rho_{F}$.

Note that if $F_{1}, F_{2}: B^{n} \longrightarrow B$ are such that $F_{2}(X)=$ $k+F_{1}(X)$ for $X \in B^{n}$ and $k \in B$, then $\Gamma_{F_{1}}=\Gamma_{F_{2}}$ because $F_{2}(X)+F_{2}(Y)=k+F_{1}(X)+k+F_{1}(Y)=$ $F_{1}(X)+F_{1}(Y)$ for every $X, Y \in B^{n}$. Therefore, it is clear that several distinct functions may share the same graph. In particular, $\Gamma_{F}=\Gamma_{F^{\prime}}$, where $F^{\prime}(X)=1+F(X)$ for $X \in B^{n}$.

Definition 2.1 Let $F: B^{n} \longrightarrow B$ be an arbitrary function (not necessarily Boolean) over the Boolean algebra $\mathcal{B}=$ $\left(B, \vee, \cdot{ }^{\prime}, 0,1\right)$.

A Boolean $\pi$-interpolation of $F$ is a family of Boolean functions $\left\{f_{\mathrm{C}} \mid \mathcal{C} \in \pi\right\}$ indexed by a partition $\pi$ of the set $B^{n}$ such that for every block $\mathcal{C}$ of $\pi, f_{\mathcal{C}}(X)=F(X)$ for every $X \in \mathcal{C}$.

An interpolation of the least cardinality is said to be $o p$ timal.

Note that every set of the form $F^{-1}(b)$ is an independent set in the graph $\Gamma_{F}$ for $b \in B$. Indeed, if $X, Y \in F^{-1}(b)$, then $F(X)+F(Y)=0 \leq(X+Y)^{\mathbf{v}}$, so there is no edge between $X$ and $Y$. Moreover, if $b, c \in B$, then the set $F^{-1}(b) \cup F^{-1}(c)$ is independent if $b+c \leq d(X, Y)$ for every $X \in F^{-1}(b)$ and $Y \in F^{-1}(c)$.

The next theorem is a generalization of Theorem 2.1 from [10]:

Theorem 2.2 For every independent set $I$ in the graph $\Gamma_{F}$ the Boolean function $f_{I}: B^{n} \longrightarrow B$ defined by

$$
f_{I}(X)=\bigvee_{Y \in I}\left(F(Y) \bigvee_{A \in\{0,1\}^{n}} X^{A} Y^{A}\right)
$$

is such that $f_{I}(X)=F(X)$ for every $X \in I$.
Proof. Suppose $X \in I$. Then,

$f_{I}(X)=F(X) \vee \bigvee_{Y \in I-\{X\}}\left(F(Y) \bigvee_{A \in\{0,1\}^{n}} X^{A} Y^{A}\right)$,

and we shall prove that $F(Y) \bigvee_{A \in\{0,1\}^{n}} X^{A} Y^{A} \leq F(X)$. Indeed, $Y \in I-\{X\}$ implies $(X, Y) \notin \rho_{F}$ because $I$ is an independent set, that is, $F(X)+$ $F(Y) \leq 1+\bigvee_{A \in\{0,1\}^{n}} X^{A} Y^{A}$. Therefore, $\bigvee_{A \in\{0,1\}^{n}} X^{A} Y^{A} \leq 1+F(X)+F(Y)=F(X)+F(Y)^{\prime}$, so $F(Y) \bigvee_{A \in\{0,1\}^{n}} X^{A} Y^{A} \leq F(Y)\left(F(X)+F(Y)^{\prime}\right)=$ $F(Y) F(X) \leq F(X)$. Thus, $f_{I}(X)=F(X)$.

Theorem 2.3 For every Boolean $\pi$-interpolation $\left\{f_{\mathrm{C}} \mid\right.$ $\mathcal{C} \in \pi\}$ of a function $F$, the partition $\pi$ is a chromatic decomposition of the graph $\Gamma_{F}$.

Proof. For every $\mathcal{C} \in \pi$ and every $X, Y \in \mathcal{C}$, we have

$$
F(X)+F(Y)=f_{\mathfrak{C}}(X)+f_{\mathfrak{C}}(Y) \leq 1+\bigvee_{A \in\{0,1\}^{n}} X^{A} Y^{A}
$$

by Theorem 2.1 of [12]. This shows that $(X, Y) \notin \rho_{F}$, which implies that $\mathcal{C}$ is an independent set. Thus, $\pi$ is a chromatic decomposition of $\Gamma_{F}$.

Corollary 2.4 There is a bijection between the Boolean $\pi$ interpolations of $F$ and the chromatic decompositions of the graph $\Gamma_{F}$. Further, if $\mu$ is a minimal chromatic decomposition of the graph $\Gamma_{F}$, then the corresponding family of Boolean functions $\left\{f_{\mathcal{C}} \mid \mathcal{C} \in \mu\right\}$ is an optimal interpolation of $F$.

Proof. This statement follows immediately from Theorems 2.2 and 2.3.

The class of functions that we discuss below serves to show that Boolean interpolations for non-Boolean functions are not unique, in general.

Definition 2.5 A function $F: B^{n} \longrightarrow B$ is $K$ evanescible, where $K$ is a subset of $B^{n}$, if $F(X)=0$ for every $X \in K$.

A characterization of Boolean $K$-evanescible functions is given below:

Theorem 2.6 Let $\mathcal{B}=\left(B, \vee, \cdot{ }^{\prime}, 0,1\right)$ be a Boolean algebra and let $K \subseteq B^{n}$. A Boolean function $g: B^{n} \longrightarrow B$ is $K$-evanescible if and only if $g(A) \leq 1+\bigvee_{Z \in K} Z^{A}$ for every $A \in\{0,1\}^{n}$.

Proof. Suppose that $g$ is $K$-evanescible and take $Z \in K$. Then, since $g(Z)=\bigvee_{A \in B^{n}} g(A) Z^{A}$, we have $g(A) Z^{A}=$ 0 , so $Z^{A} \leq 1+g(A)$. Consequently, $\bigvee_{Z \in K} Z^{A} \leq 1+$ $g(A)$, so $g(A) \leq 1+\bigvee_{Z \in K} Z^{A}$. 
Conversely, suppose that $g(A) \leq 1+\bigvee_{Z \in K} Z^{A}$ for every $A \in\{0,1\}^{n}$. Then, since $g$ is a Boolean function we can write for $X \in K$ :

$$
\begin{aligned}
g(X) & =\bigvee_{A \in\{0,1\}^{n}} g(A) X^{A} \\
& \leq \bigvee_{A \in\{0,1\}^{n}}\left(1+\bigvee_{Z \in K} Z^{A}\right) X^{A} \\
& =\bigvee_{A \in\{0,1\}^{n}}\left(X^{A}+\bigvee_{Z \in K} X^{A} Z^{A}\right)=0,
\end{aligned}
$$

because $X \in K$ implies $\bigvee_{Z \in K} X^{A} Z^{A}=X^{A}$, which shows that $g$ is $K$-evanescible.

Note that if $I$ is an independent set in the graph $\Gamma_{F}$ and $f_{1}, f_{2}$ are two Boolean functions that interpolate $F$ on $I$, then $f_{1}+f_{2}$ is a Boolean $I$-evanescible function. Thus, a Boolean interpolant of $F$ for an independent set $I$ of $\Gamma_{F}$ is unique if $\bigvee_{Z \in I} Z^{A}=1$ for every $A \in\{0,1\}^{n}$.

Theorem 2.7 Let $F: B^{n} \longrightarrow B$ be a function and let $I$ be an independent set in $\Gamma_{F}$. For every Boolean interpolating function $g: B^{n} \longrightarrow B$ for $F$ on $I$ we have $f_{I} \leq g$, where $f_{I}$ is the Boolean function defined in Theorem 2.2.

Proof. Since $g$ is an interpolating Boolean function for $F$ we can write $g(X)=\bigvee_{C \in\{0,1\}^{n}} g(C) X^{C}=F(X)$ for every $X \in I$. Therefore,

$$
F(X) X^{A}=\bigvee_{C \in\{0,1\}^{n}} g(C) X^{C} X^{A}=g(A) X^{A} \leq g(A)
$$

for every $X \in I$. Consequently, $\bigvee_{X \in I} F(X) X^{A} \leq g(A)$. Since

$$
f_{I}(A)=\bigvee_{X \in I} F(X) X^{A}
$$

for every $A \in\{0,1\}^{n}$ it follows that $f_{I}(A) \leq g(A)$ for every $A \in\{0,1\}^{n}$, so $f_{I} \leq g$.

\section{Boolean and Isolated Vertices in Graphs of Functions}

In [12] we extended the classes of upper and lower semi-Boolean functions of one-variable introduced in [9] by introducing the notion of $A$-Boolean function for $A \in$ $\{0,1\}^{n}$.

For $A \in\{0,1\}^{n}$ we say that $F: B^{n} \longrightarrow B$ is an $A$ Boolean function if $F(X)+F(Y) \leq 1+X^{A} Y^{A}$ for every $X, Y \in B^{n}$. The next lemma will allow us to formulate another definition of $A$-Boolean functions.

Lemma 3.1 Let $\mathcal{B}=\left(B, \vee, \cdot{ }^{\prime}, 0,1\right)$ be a Boolean algebra, $F: B^{n} \longrightarrow B, X \in B^{n}$ and $A \in\{0,1\}^{n}$. The following conditions are equivalent:
1. $X^{A} F(X)=X^{A} F(A)$;

2. $F(X)+F(A) \leq 1+X^{A}$;

3. $F(X)+F(Y) \leq 1+X^{A} Y^{A}$ for every $Y \in B^{n}$.

Proof. (1) implies (2): We have $X^{A}(F(X)+F(A))=$ $X^{A} F(X)+X^{A} F(A)=0$.

(2) implies (3): By the Verification Theorem with respect to $Y$ because for $Y=A$ condition (3) reduces to (2), while for $Y \in\{0,1\}^{n}-\{A\}$, condition (3) reduces to the identity $F(X)+F(Y) \leq 1$.

(3) implies (1): By condition (3), choosing $Y=A$ we have $F(X)+F(A) \leq 1+X^{A} A^{A}=1+X^{A}$. Thus, $X^{A} F(X)+X^{A} F(A) \leq X^{A}+X^{A}=0$, which implies $X^{A} F(X)+X^{A} F(A)=0$.

Definition 3.2 Let $\mathcal{B}=\left(B, \vee, \cdot{ }^{\prime}, 0,1\right)$ be a Boolean algebra. A function $F: B^{n} \longrightarrow B$ is locally Boolean at point $X \in B^{n}$ if

$$
F(X)=\bigvee_{A \in\{0,1\}^{n}} F(A) X^{A} .
$$

If $F$ is locally Boolean at $X$, then we say that $X$ is a Boolean point of $F$.

It is easy to see that all points $C \in\{0,1\}^{n}$ are Boolean points for $F$.

Theorem 3.3 Let $F: B^{n} \longrightarrow B$ be a function and let $X \in B^{n}$, where $\mathcal{B}=\left(B, \vee, \cdot{ }^{\prime}, 0,1\right)$ is a Boolean algebra.

$X \in B^{n}$ is a Boolean point of $F$ if and only if any of the equivalent conditions of Lemma 3.1 are satisfied for every $A \in\{0,1\}^{n}$.

Proof. Suppose that $X$ is a Boolean point of $F$. By multiplying both sides of the equality (2) by $X^{A}$ we have $X^{A} F(X)=X^{A} F(A)$, which is the first condition of Lemma 3.1.

Conversely, suppose that $X^{A} F(X)=X^{A} F(A)$ is satisfied for every $A \in\{0,1\}^{n}$. Then, by taking the join of these equalities and using Part 1 of Lemma 1.1 we have:

$$
F(X)=\bigvee_{A \in\{0,1\}^{n}} X^{A} F(X)=\bigvee_{A \in\{0,1\}^{n}} X^{A} F(A),
$$

which shows that $X$ is a Boolean point of $F$.

Note that a point $X \in B^{n}$ is isolated in the graph $\Gamma_{F}$ if and only if there is no edge $(X, Y)$ for any $Y \in B^{n}$, or, equivalently, if

$$
F(X)+F(Y) \leq(X+Y)^{\vee} .
$$

for every $Y \in B^{n}-\{X\}$. Therefore, the graph $\Gamma_{F}$ of any Boolean function is totally disconnected. 
Lemma 3.4 The following conditions are equivalent:

$$
\begin{aligned}
& \text { 1. } F(X)+F(Y) \leq(X+Y)^{\vee} \text { for every } Y \in B^{n} ; \\
& \text { 2. } F(X)+F(Y) \leq 1+\bigvee_{A \in\{0,1\}^{n}} X^{A} Y^{A} \text { for every } \\
& Y \in B^{n} ; \\
& \text { 3. } F(X)+F(Y) \leq 1+X^{A} Y^{A} \text { for every } Y \in B^{n} \text { and } \\
& \text { every } A \in\{0,1\}^{n} .
\end{aligned}
$$

Proof. The equivalence of the first two conditions follows immediately from Part 2 of Lemma 1.1. Using the DeMorgan Law

$$
1+\bigvee_{A \in\{0,1\}^{n}} X^{A} Y^{A}=\bigwedge_{A \in\{0,1\}^{n}}\left(1+X^{A} Y^{A}\right)
$$

we obtain the equivalence of the last two conditions.

Theorem 3.5 Every isolated point of a function $F$ is a Boolean point of the function.

Proof. Suppose that $X \in B^{n}$ is an isolated point of $F$. Then, by Lemma 3.4 we have $F(X)+F(Y) \leq 1+$ $\bigvee_{A \in\{0,1\}^{n}} X^{A} Y^{A}$ for every $Y \in B^{n}$. In particular, taking $Y=A \in\{0,1\}^{n}$ we have

$$
F(X)+F(A) \leq 1+X^{A}
$$

for every $A \in\{0,1\}^{n}$, which is one of the equivalent conditions of Lemma 3.1.

If $B$ is a finite Boolean algebra that has $k$ atoms, then $\left|B^{n}\right|=2^{k n}$. Thus, it is easier to test whether $X \in B^{n}$ is a Boolean point for a function $F$ than to test whether $X$ is an isolated point in $\Gamma_{F}\left(2^{k}\right.$ vs. $\left.2^{k n}\right)$ tests), especially if $B$ has a large number of atoms. In computing the graph $\Gamma_{F}$, we begin by identifying the Boolean points and, then, determine which of these are isolated points.

Example 3.6 Consider the 4-element Boolean algebra $\mathcal{B}=$ $\left(\left\{0,1, c, c^{\prime}\right\}, \vee, \cdot{ }^{\prime}, 0,1\right)$ and a function $F: B \longrightarrow B$. We saw that 0,1 are Boolean points. We claim 0 is an isolated point in the graph $\Gamma_{F}$ if and only if $F(0)=c F\left(c^{\prime}\right)+c^{\prime} F(c)$; similarly, 1 is an isolated point in $\Gamma_{F}$ if $F(1)=c F(c)+$ $c^{\prime} F\left(c^{\prime}\right)$.

An element $a$ is an isolated point in $\Gamma_{F}$ if and only if the all of the following conditions are satisfied:

$$
\begin{aligned}
F(a)+F(0) & \leq a \\
F(a)+F(1) & \leq a+1, \\
F(a)+F(c) & \leq a+c, \\
F(a)+F\left(c^{\prime}\right) & \leq a+c^{\prime} .
\end{aligned}
$$

It is clear that $a=0$ trivially satisfies the first two conditions. Thus, 0 is an isolated point if and only if $F(0)+$

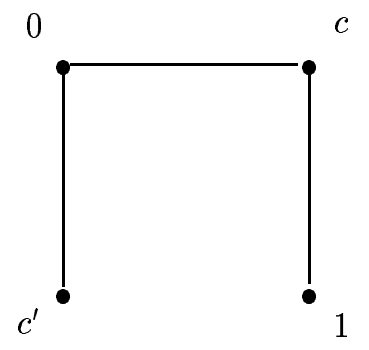

Figure 1. The Graph $\Gamma_{F}$

$F(c) \leq c$ and $F(0)+F\left(c^{\prime}\right) \leq c^{\prime}$. These conditions imply $F(0) c^{\prime}=F(c) c^{\prime}$ and $F(0) c=F\left(c^{\prime}\right) c$, respectively. Adding the last two equalities yields $F(0)=c F\left(c^{\prime}\right)+$ $c^{\prime} F(c)$.

Conversely, if $F(0)=c F\left(c^{\prime}\right)+c^{\prime} F(c)$, then $F(0)+$ $F(c)=c F\left(c^{\prime}\right)+c F(c) \leq c$ and $F(0)+F\left(c^{\prime}\right)=c^{\prime} F(c)+$ $c^{\prime} F\left(c^{\prime}\right) \leq c^{\prime}$, which shows that 0 is an isolated point. The argument for 1 is similar.

It is not difficult to see that $c$ is an isolated point if and only if $F(c)=c F(1)+c^{\prime} F(0)$, hence $c^{\prime}$ is such a point if $F\left(c^{\prime}\right)=c F(0)+c^{\prime} F(1)$. Further, if both $c$ and $c^{\prime}$ are isolated, then so are 0 and 1 .

Consider, for example, the function $F$ defined by $F(0)=0, F(c)=c^{\prime}, F\left(c^{\prime}\right)=F(1)=1$. None of the vertices of $\Gamma_{F}$ is isolated. Indeed, the graph of this function is shown in Figure 1.

Since the graph is not the totally disconnected graph, $F$ is not a Boolean function; however, the chromatic number of this graph is 2 , because the sets $\mathcal{C}_{0}=\{0,1\}$ and $\mathcal{C}_{1}=\left\{c, c^{\prime}\right\}$ are maximal independent sets. The Boolean interpolating functions are:

$$
\begin{aligned}
f_{\mathcal{C}_{0}}(x) & =F(0)\left(x^{0} 0^{0} \vee x^{1} 0^{1}\right) \vee F(1)\left(x^{0} 1^{0} \vee x^{1} 1^{1}\right)=x \\
f_{\mathcal{C}_{1}}(x) & =F(c)\left(x^{0} c^{0} \vee x^{1} c^{1}\right) \vee F\left(c^{\prime}\right)\left(x^{0}\left(c^{\prime}\right)^{0} \vee x^{1}\left(c^{\prime}\right)^{1}\right) \\
& =c^{\prime}\left(x^{\prime} c^{\prime} \vee x c\right) \vee 1\left(x^{\prime} c \vee x c^{\prime}\right) \\
& =x^{\prime} c^{\prime} \vee x^{\prime} c \vee x c^{\prime}=x^{\prime} \vee x c^{\prime}=x^{\prime} \vee c^{\prime}
\end{aligned}
$$

Note that for both $\mathcal{C}_{0}=\{0,1\}$ and $\mathcal{C}_{1}=\left\{c, c^{\prime}\right\}$ the interpolating functions are unique.

Example 3.7 Consider the binary function $F: B^{2} \longrightarrow B$, where $B$ is the four-element Boolean algebra introduced in Example 3.6 and $F$ is the binary function specified by the 


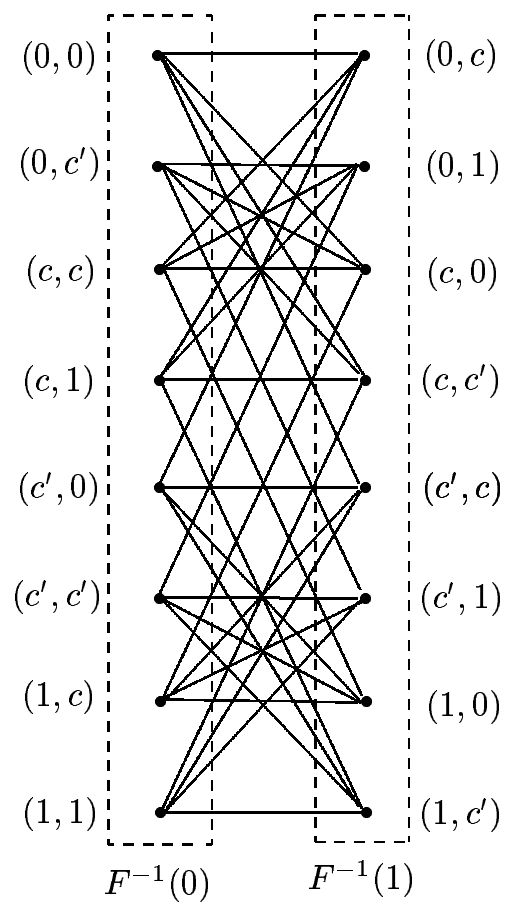

Figure 2. $\Gamma_{F}$ for the function $F: B^{2} \longrightarrow B$ of Example 3.7

following table:

\begin{tabular}{|c|cccc|}
\hline$F(x, y)$ & 0 & $c$ & $c^{\prime}$ & 1 \\
\hline 0 & 0 & 1 & 0 & 1 \\
$c$ & 1 & 0 & 1 & 0 \\
$c^{\prime}$ & 0 & 1 & 0 & 1 \\
1 & 1 & 0 & 1 & 0 \\
\hline
\end{tabular}

The graph $\Gamma_{F}$ is shown in Figure 2. The Boolean functions that correspond to the maximal independent sets $\mathrm{C}_{0}=$ $F^{-1}(0)$ and $\mathcal{C}_{1}=F^{-1}(1)$ are $f_{\mathcal{C}_{0}}(X)=0$ and

$$
\begin{aligned}
f_{\mathcal{C}_{1}}(X) & =\bigvee_{F(Y)=1} \bigvee_{A \in\{0,1\}^{n}} X^{A} Y^{A} \\
& =\bigvee_{A \in\{0,1\}^{n}} \bigvee_{F(Y)=1} X^{A} Y^{A} \\
& =\bigvee_{A \in\{0,1\}^{n}} X^{A} \bigvee_{F(Y)=1} Y^{A} .
\end{aligned}
$$

A direct verification shows that

$$
\begin{aligned}
& \bigvee_{F(Y)=1} Y^{(0,0)}=c^{\prime} \\
& \bigvee_{F(Y)=1} Y^{(0,1)}=1 \\
& \bigvee_{F(Y)=1} Y^{(1,0)}=1 \\
& \bigvee_{F(Y)=1} Y^{(1,1)}=c^{\prime} .
\end{aligned}
$$

Thus,

$$
f_{\mathrm{C}_{1}}(X)=X^{(0,0)} c^{\prime} \vee X^{(0,1)} \vee X^{(1,0)} \vee X^{(1,1)} c^{\prime} .
$$

\section{Conclusions and Open Problems}

The graph $\Gamma_{F}$ of an arbitrary function $F: B^{n} \longrightarrow B$ over a Boolean algebra defined in this paper is a tool for determining Boolean interpolations using the independent sets in such graphs. An algorithm that finds maximal independent sets using Boolean techniques is given in [7].

If $K$ is a maximal independent set and $X \in B^{n}$ denote by $b_{X}^{K}$ a Boolean variable, where

$$
b_{X}^{K}= \begin{cases}1 & \text { if } X \in K \\ 0 & \text { otherwise }\end{cases}
$$

Then, the $K$ is a maximal independent set in $\Gamma_{F}$ if the family $\left\{b_{X}^{K}\right\}$ satisfies the system of Boolean equations

$$
b_{X}^{K}=\prod\left\{\left(b_{Y}^{K}\right)^{\prime} \mid F(X)+F(Y) \not \subset(X+Y)^{\mathbf{v}}\right\}
$$

for $X \in B^{n}$. Such systems can be solved using a branching technique, as discussed in [7]. We are now developing a specialized algorithm that begins with the specification of the non-Boolean function in a tabular form and generates the maximal independent sets of the graph $\Gamma_{F}$.

Graphs of special classes of functions such as generalized Boolean functions (see [13, 14, 15]), or chain-valued functions, etc. should also be investigated.

\section{Acknowledgement}

The authors wish to acknowledge the support received through a grant from the National Research Council COBASE program. 


\section{References}

[1] T. Aoki. Dreams for new device-based superchips. In Proc. 23rd IEEE Int. Symp. Multiple-Valued Logic, pages 140149, 1993.

[2] T. Aoki and T. Higuchi. Impact of interconnection-free biomolecular computing. In Proc.23rd IEEE Int. Symp. Multiple-Valued Logic, pages 271-276, 1993.

[3] T. Aoki, M. Kameyama, and T. Higuchi. Design of a highly parallel set logic network based on a bio-device model. In Proc. of the 19th Symposium for Multiple-Valued Logic, pages 360-367, 1989.

[4] T. Aoki, M. Kameyama, and T. Higuchi. Interconnectionfree set logic network based on a bio-device model. IEEE Letters, 26:1015-1016, 1990.

[5] T. Aoki, M. Kameyama, and T. Higuchi. Design of interconnection-free biomolecular computing system. In Proc.21st IEEE Int. Symp. Multiple-Valued Logic, pages 173-180, 1991.

[6] T. Aoki, M. Kameyama, and T. Higuchi. Interconnectionfree biomolecular computing. IEEE Computer, 25:41-50, 1992.

[7] P. L. Hammer and S. Rudeanu. Boolean Methods in Operation Research and Related Areas. Springer Verlag, Berlin, 1967.

[8] M. Kameyama and T. Higuchi. Prospects of multiplevalued bio-information processing systems. In Proc. of the 18th Symposium for Multiple-Valued Logic, pages 237-242, 1988.

[9] R. Melter and S. Rudeanu. Functions characterized by functional Boolean equations. Colloquia Mathematica Societatis Janos Bolyai, 33:637-650, 1980.

[10] C. Reischer and D. A. Simovici. On the implementation of set-valued non-Boolean switching functions. In Proceedings of the 21st International Symposium for MultipleValued Logic, pages 166-172, 1991.

[11] S. Rudeanu. Boolean Functions and Equations. NorthHolland/American Elsevier, Amsterdam, 1974.

[12] D. A. Simovici. Several remarks on non-Boolean functions over Boolean algebra. In Proceedings of the 33rd International Symposium for Multiple-Valued Logic, 2003.

[13] N. Ţăndăreanu. On generalized Boolean functions. I. Discrete Mathematics, 34:293-299, 1981.

[14] N. Ţăndăreanu. On generalized Boolean functions. II. Discrete Mathematics, 40:277-284, 1982.

[15] N. Ţăndăreanu. On generalized Boolean functions. III. The case $A=\{0,1\}$. Discrete Mathematics, 52:269-277, 1984. 